\title{
Dénutrition cancéreuse et inflammation : effet des acides gras polyinsaturés n-3
}

\author{
Jean-François DUMAS ${ }^{1,2}$ \\ Charles COUET ${ }^{1,2,3}$ \\ Philippe BOUGNOUX ${ }^{1,2,3}$ \\ Stéphane SERVAIS ${ }^{1,2,4}$ \\ 1 Inserm U921, \\ nutrition, croissance et cancer, 37032 \\ Tours, France \\ <jean-francois.dumas@univ-tours.fr> \\ 2 Université François-Rabelais, faculté \\ de médecine, bâtiment Dutrochet, 10, \\ boulevard Tonnellé, 37032 Tours, \\ France \\ ${ }^{3} \mathrm{CHRU}$ de Bretonneau, \\ 37044 Tours, France \\ 4 IUT, \\ 37082 Tours, France
}

De part sa complexité et son origine multifactorielle, lutter contre la dénutrition chez le patient atteint de cancer est difficile et doit associer des approches médicamenteuses et nutritionnelles. L'objectif de cet article est de faire le point sur la dénutrition cancéreuse, le rôle joué par I'inflammation et sur les effets bénéfiques des acides gras polyinsaturés à longue chaîne de la série $n-3$ (AGPI n-3).

\section{La dénutrition cancéreuse, un syndrome multifactoriel complexe}

La dénutrition est un processus dynamique très fréquent au cours du cancer. Elle affecte 31 à $87 \%$ des patients au moment du diagnostic du cancer et, dans sa forme sévère (appelée cachexie), contribue directement à environ $20 \%$ des décès chez le patient cancéreux (Dewys et al., 1980). Si cette

\begin{abstract}
Undernutrition is frequently seen in cancer and is considered as a bad prognostic factor. The mechanisms involved in this complex syndrome are not fully understood. Systemic inflammation plays an important role in the occurrence and/or development of cancer-associated undernutrition. Although it is essential to counteract cancer-associated undernutrition, modalities for nutritional treatment are still discussed. It has been shown that nutritional supplementation with $n-3$ polyunsaturated fatty acid could be beneficial in patients.
\end{abstract}

Key words: cachexia, cytokines, metabolism, mitochondria

dénutrition n'est pas toujours létale, elle conduit très souvent à une impasse thérapeutique. En effet, la dénutrition cancéreuse diminue de façon importante la qualité de vie en altérant les capacités fonctionnelles et l'autonomie des malades qui, par conséquent, supportent de plus en plus difficilement les traitements anticancéreux (chimiothérapie, radiothérapie...). Le degré de dénutrition est un indice de mauvais pronostic. II est inversement corrélé à la survie des patients. La prévention et le traitement de la dénutrition cancéreuse sont donc des objectifs fondamentaux de la lutte contre le cancer. Malheureusement, il n'existe pas actuellement de traitement spécifique permettant de lutter efficacement contre la dénutrition du patient cancéreux. Les traitements nutritionnels, quant à eux, n'ont qu'une efficacité modeste ( $\mathrm{Ng}$ and Lowry, 1991).

La dénutrition cancéreuse est un syndrome multifactoriel qui se caractérise par une balance énergétique négative et une diminution des masses musculaire et adipeuse (Argiles et al., 2010; Muscaritoli et al., 2010). Le déficit de la balance énergétique s'explique par une diminution des apports (anorexie) et une augmentation des dépenses énergétiques (hypermétabolisme). Tous les mécanismes impliqués dans la survenue de la dénutrition ne sont pas encore totalement élucidés (Tisdale, 2009). L'anorexie est très présente ainsi que de nombreuses altérations métaboliques (stimulation des catabolismes des glucides, des lipides et des protéines) dont une partie explique l'augmentation de la dépense énergétique basale. Ces altérations qui touchent un grand nombre de tissus et d'organes (foie, tissu adipeux et muscle squelettique...) sont déclenchées par la libération de différents facteurs par l'hôte et la tumeur, des altérations hormonales et une inflammation systémique (libération de cytokines pro-inflammatoires : interleukine(IL)-1 $\beta$, IL-6 et tumor necrosis factor (TNF- $\alpha$, par exemple) (Argiles et al., 2010; Muscaritoli et al., 2010).

Parmi les altérations métaboliques associées à la dénutrition cancéreuse, il semble que la présence d'un dysfonctionnement du métabolisme énergétique mitochondrial puisse jouer un rôle. II a ainsi été montré que chez des rats cancéreux (carcinome mammaire) qui perdaient du poids, l'activité des complexes de la chaîne respiratoire, au sein de mitochondries isolées de foie et des glandes mammaires, était diminuée (Perumal et al., 2005). À l'inverse, la perte de poids était réduite dans le groupe de rats cancéreux traités avec un supplément vitaminique (riboflavine, coenzyme Q10) chez qui la diminution

Pour citer cet article : Dumas JF, Couet C, Bougnoux P, Servais S. Dénutrition cancéreuse et inflammation : effet des acides gras polyinsaturés n-3. OCL 2011 ; 18(1) : 34-38. doi : 10.1684/ocl.2011.0362 
de l'activité des complexes enzymatiques n'était pas présente (Perumal et al., 2005). La mitochondrie, organite cellulaire, est la principale source d'énergie pour l'organisme puisque près de $90 \%$ de I'ATP sont produits au cours du processus de la phosphorylation oxydative (Rolfe and Brown, 1997). Dans ce processus, l'énergie libérée des molécules organiques provenant des aliments est transformée en un gradient électrochimique de part et d'autre de la membrane interne de la mitochondrie. $\mathrm{Au}$ sein de la chaîne respiratoire, la cascade de réactions d'oxydo-réduction nécessaire aboutie à la consommation d'oxygène (I'accepteur final d'électron). L'énergie du gradient électrochimique est ensuite utilisée pour permettre la phosphorylation de I'ADP en ATP par I'ATP synthase. II existe donc un couplage entre la consommation d'oxygène par la chaîne respiratoire mitochondriale et la synthèse d'ATP. Cependant ce couplage (ou efficacité de la phosphorylation oxydative) n'est pas parfait et toute l'énergie libérée des aliments n'est pas convertie en ATP mais une partie est perdue sous la forme de chaleur. Ainsi, dans une situation où l'efficacité de la synthèse d'ATP est abaissée sans diminution concomitante de la demande énergétique par la cellule, I'utilisation des nutriments augmentera. Une telle situation sera d'autant plus délétère sur le poids, la masse grasse et la masse maigre que les apports alimentaires seront réduits obligeant l'organisme à puiser sur ses réserves énergétiques. Très récemment nous avons montré, dans un modèle murin de dénutrition cancéreuse, que l'efficacité de synthèse de I'ATP au sein des mitochondries hépatiques est fortement réduite (-24\%) chez les rats dénutris (Dumas et al., 2010a; Dumas et al., 2010b). En conséquence, les mitochondries doivent consommer plus de nutriments pour satisfaire la demande cellulaire en ATP. Cela pourrait participer à l'augmentation de la dépense énergétique basale et donc à la perte de poids des rats cancéreux (figure 1).

\section{Inflammation et dénutrition cancéreuse}

La présence d'une inflammation chronique systémique qui inclut la libération de cytokines pro-inflammatoires (IL-1 $\beta$, IL-6, TNF- $\alpha .$. ) est une caractéristique forte de la dénutrition cancéreuse. Ainsi dans une étude réalisée sur dix patients sains et un groupe de 43 patients cancéreux (prostate) comprenant 17 patients non dénutris et 26 patients souffrant de dénutrition, il a été montré que les concentrations sériques de TNF$\alpha$, d'IL-6 et d'IL-8 étaient spécifiquement augmentées chez les patients

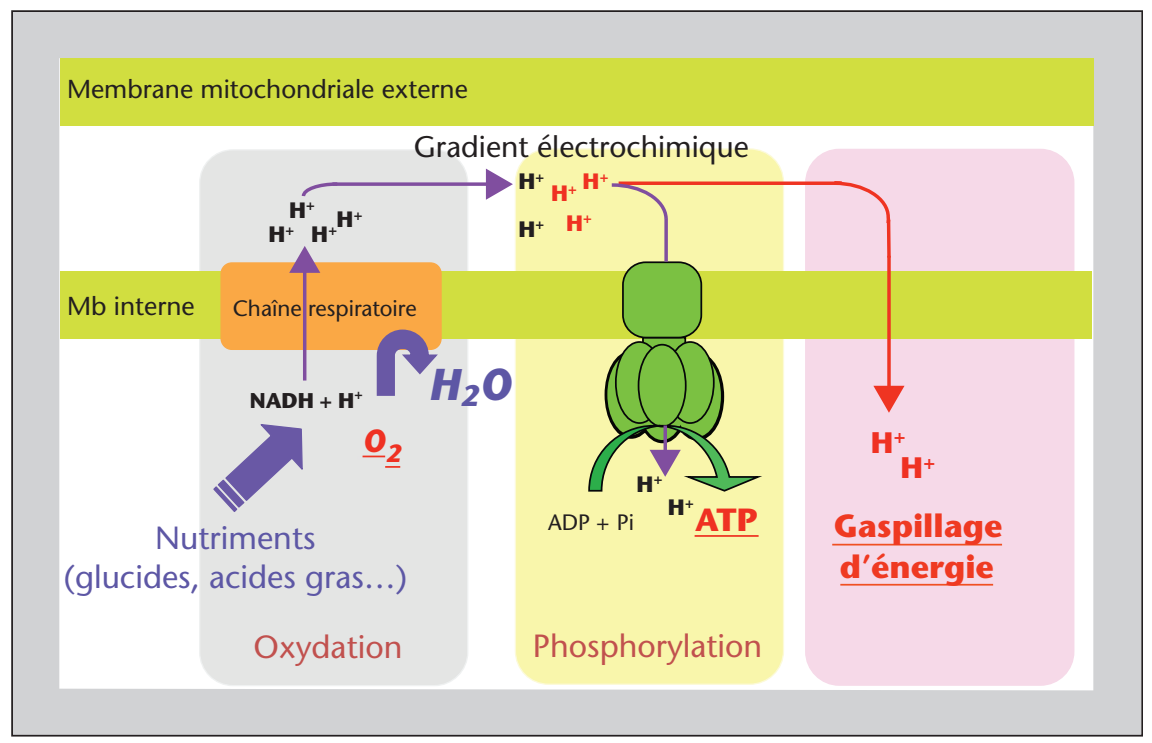

Figure 1. Dans les mitochondries hépatiques des rats cancéreux dénutris, la part du gradient électrochimique non utilisé pour la synthèse d'ATP (gaspillage d'énergie) est plus importante que chez les rats sains. Pour compenser la consommation d'oxygène et donc de nutriments est augmentée. Cette demande supplémentaire en énergie (nutriments) pourrait contribuer à I'augmentation du métabolisme de base. cancéreux souffrant de dénutrition en comparaison de ceux non dénutris (Pfitzenmaier et al., 2003). Les cytokines impliquées dans cette réponse inflammatoire peuvent être produites par I'hôte en réponse à la tumeur et par la tumeur elle-même. Dans le contexte de la dénutrition cancéreuse, le syndrome inflammatoire par lui-même est capable d'avoir un impact négatif sur le pronostic vital des patients cancéreux. C'est ce qui a été montré dans une étude réalisée sur 106 patients souffrant d'un cancer des poumons inopérable, où les auteurs en classant l'ensemble des patients en trois catégories selon leur concentration sérique en C-Réactive Protéine (CRP) ont mis en évidence que les patients chez qui la réponse inflammatoire était la plus marquée étaient ceux qui perdaient le plus de poids et qui avaient le taux de survie le plus faible (Scott et al., 2002). Par ailleurs, il a été démontré que des traitements anti-inflammatoires (indométhacine ou prednisolone ou celecoxib) étaient capables d'améliorer le taux de survie de patients cancéreux dénutris même si les effets bénéfiques concernant la préservation de la masse musculaire n'étaient pas toujours présents et n'impliqueraient pas toujours des changements de la concentration circulante des cytokines (Lundholm et al., 1994; Lundholm et al., 2004; Lai et al., 2008; Mantovani et al., 2010).

De nombreuses études animales ont permis de mettre en évidence un lien direct entre les cytokines pro-inflammatoires et la dénutrition cancéreuse. Par exemple, chez des souris souffrant de dénutrition cancéreuse (modèle sous cutané colon 26-adenocarcinoma), il a été montré que suite à la résection de la tumeur, les animaux retrouvaient un poids corporel et un taux sérique d'IL-6 comparables à des animaux sains (Strassmann et al., 1992). Parallèlement, lorsqu'à l'injection des cellules tumorales, les souris étaient également traitées avec un anti-IL-6, ces dernières ne développaient pas de dénutrition (Strassmann et al., 1992). Toutefois, aucun effet bénéfique n'était retrouvé suite à I'utilisation d'anti-TNF- $\alpha$. Ce résultat montre que les cytokines impliquées dans la dénutrition associée au cancer dépendent évidemment du type de tumeur. Pourtant aucune ne semble agir seule. En effet, il a été rapporté que l'injection d'un clone particulier, dérivé 
de cellules de colon 26-adenocarcinoma, chez la souris était capable de mimer l'augmentation de la concentration sérique d'IL-6 retrouvée suite à l'injection de cellules de colon 26adenocarcinoma sans toutefois reproduire de dénutrition cancéreuse (Soda et al., 1994).

Concernant les mécanismes impliqués dans la dénutrition cancéreuse, il a été rapporté que les cytokines étaient capables d'agir sur un grand nombre d'entre eux. Ainsi, I'injection intrapéritonéale de TNF- $\alpha$ ou d'interferon (IFN)- $\gamma$ est capable de mimer l'anorexie (Langstein et al., 1991). Les cytokines influencent la prise alimentaire, d'une part, en diminuant la libération des médiateurs orexigéniques tels que le neuropeptide $Y$ et, d'autre part, en stimulant la libération des médiateurs anorexigéniques comme le corticotrophin releasing factor (CRF) (Morley et al., 2006). L'inflammation semble aussi participer à la diminution de la masse adipeuse (Lopez-Soriano et al., 1997). Il a par exemple été montré que le TNF- $\alpha$ pouvait stimuler la lipolyse d'adipocytes humains (Zhang et al., 2002). La fonte musculaire peut $s^{\prime}$ expliquer soit par une stimulation de la dégradation des protéines soit par une diminution de la synthèse protéique ou les deux. Lors de la dénutrition cancéreuse, les études font surtout mention d'un hypercatabolisme protéique qui impliquerait l'ensemble des systèmes protéolytiques et notamment les voies lysosomales et ATP-ubiquitineprotéasome dépendante (Baracos, 2000). De plus, dans un grand nombre de modèles de dénutrition cancéreuse, cet hypercatabolisme serait, en partie, sous le contrôle des cytokines (Baracos, 2000). Dans le modèle Yoshida AH-130, lorsque les rats cancéreux sont également traités avec un inhibiteur de la synthèse du TNF- $\alpha$ ou un bloqueur de l'action périphérique de certaines cytokines (notamment IL- 6 et TNF- $\alpha$ ), la fonte musculaire est prévenue et les activités des voies protéolytiques ATPubiquitine-protéasome dépendante et calpaine-dépendente sont réduites (Costelli et al., 2002). II a par ailleurs été montré que I'injection intrapéritonéale d'IL-1, de TNF- $\alpha$ ou d'IFN- $\gamma$ était capable de stimuler l'expression de gènes du système ATP-ubiquitineprotéasome dépendent (Llovera et al., 1998). Récemment, le rôle que pourrait jouer la myostatine, facteur de croissance qui limite le développement musculaire, dans la dénutrition cancéreuse et plus particulièrement dans la fonte musculaire associée, a été mis en évidence. Ainsi, dans le modèle de dénutrition cancéreuse Yoshida $\mathrm{AH}-130$, il a été montré une surexpression de la myostatine dans le gastrocnemius des rats cancéreux dénutris et que cette surexpression n'était pas présente si les rats cancéreux étaient traités avec un inhibiteur de la synthèse du TNF- $\alpha$ (Costelli et al., 2008). D'un autre côté, il a été montré que la myostatine jouait bien un rôle dans la dénutrition cancéreuse mais que ce mécanisme n'était pas forcément sous le contrôle de l'inflammation, en tout cas concernant les cytokines IL-1, IL-6 et TNF- $\alpha$ (Zhou et al., 2010). Finalement, il n'est pas exclu que les cytokines puissent aussi jouer un rôle dans la dénutrition cancéreuse en participant au dysfonctionnement du métabolisme énergétique mitochondrial. Bien qu'il n'y ait pas encore de preuves directes, il a par exemple été montré que le TNF- $\alpha$ et I'IL-1 $\beta$ étaient capables d'altérer in vitro le métabolisme énergétique mitochondrial (Tatsumi et al., 2000; Busquets et al., 2003; Samavati et al., 2008).

\section{Effet des AGPI n-3 sur la dénutrition cancéreuse}

Parmi les possibilités de lutte contre la dénutrition cancéreuse, une supplémentation en AGPI n-3 à longue chaîne et notamment en acides eicosapentaénoïque (EPA) et docosahexaénoïque ( $\mathrm{DHA}$ ) semble prometteuse. Ainsi, bien que cela soit discuté (Dewey et al., 2007), il semble qu'une supplémentation orale en AGPI n-3 permette de stabiliser le poids, d'améliorer les paramètres nutritionnels et la qualité de vie chez certains patients dénutris atteints de cancer (Colomer et al., 2007). Les divergences entre les études peuvent s'expliquer par le type de cancer considéré, le stade d'avancement de la dénutrition du patient, la nature et la capacité d'incorporation de ces acides gras dans les tissus (Bougnoux et al., 2009) et la durée du traitement nutritionnel. De manière moins discutée, les résultats obtenus sur des modèles animaux de dénutrition cancéreuse montrent que les AGPI n-3 limitent les pertes tissulaires musculaire et adipeuse et augmentent la survie des animaux (Jho et al., 2002; Togni et al., 2003). Par exemple, nous avons récemment démontré, dans un modèle murin de dénutrition cancéreuse (induite par une carcinose péritonéale) que des AGPI n-3 d'origine marine (régime à base d'huile de poisson : $17 \%$ de DHA et $8 \%$ d'EPA), présents dans I'alimentation dès six semaines avant l'induction du cancer, retardent de façon très importante l'apparition de l'anorexie (figure 2) (Dumas et al., 2010b).

Les mécanismes qui pourraient expliquer I'effet bénéfique des AGPI n-3 dans la dénutrition cancéreuse sont nombreux et ne sont pas encore totalement

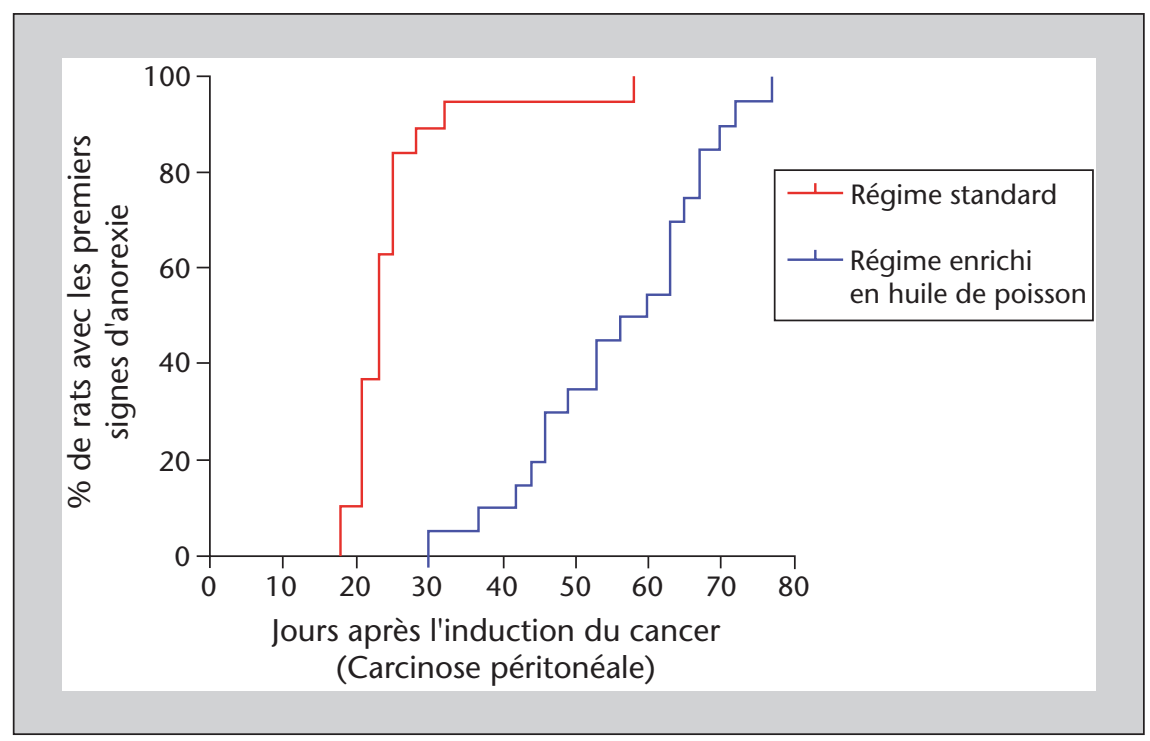

Figure 2. Effet d'un régime enrichi en huile de poisson (riche en AGPI $n$-3) sur la cinétique d'apparition de la dénutrition dans un modèle de rat cancéreux. 
élucidés. Certaines études montrent que les AGPI $n-3$ réduisent la croissance tumorale (Zhou et al., 2010; Togni et al., 2003). D'autres résultats suggèrent plutôt que I'effet des AGPI n-3 sur la dénutrition serait indépendant de celui sur la croissance de la tumeur et interfèrerait donc directement avec les mécanismes cellulaires et moléculaires impliqués dans la dénutrition induite par le cancer (Dumas et al., 2010b; Jho et al., 2002). II a ainsi été montré que I'EPA atténue la perte de masse musculaire chez des souris dénutries (tumeur MAC16) en inhibant l'activité de protéolyse ATP-ubiquitine-protéasome dépendante (Whitehouse et al., 2001). II a également été montré qu'une alimentation supplémentée en AGPI n-3 permettait de maintenir une prise alimentaire normale (maintien du nombre et de la taille des repas) chez des rats, probablement en jouant sur les mécanismes de régulation de la prise alimentaire et ce en modulant l'intensité de la réponse inflammatoire (Ramos et al., 2004). En effet, les AGPI n-3 sont connus pour leurs propriétés anti-inflammatoires. Cela implique :

- I'inhibition de la production des eicosanoides dérivés de l'acide arachidonique ;

- l'activation de la production des résolvines;

- I'inhibition directe de la production de cytokines pro-inflammatoires (Calder, 2009).

Il a ainsi été rapporté qu'un régime riche en AGPI n-3 était capable de diminuer la production de cytokines pro-inflammatoires telles que IL- 6 et TNF- $\alpha$ (Wigmore et al., 1996; Wigmore et al., 1997). En diminuant la production de cytokines pro-inflammatoires, il est donc attendu que les AGPI n-3 puissent lutter contre la dénutrition cancéreuse en modulant l'ensemble des mécanismes stimulés par les cytokines. Il a ainsi été démontré que les AGPI n-3 interagissaient avec IL1 sur la prise alimentaire puisque contrairement à des rats nourris avec une alimentation standard ou riche en acide linoléique, chez des rats nourris avec une alimentation enrichie en huile de poisson (17\% EPA et $12 \%$ DHA) I'injection intraveineuse d'IL-1 recombinante $n^{\prime}$ induisait pas de diminution significative de la prise alimentaire (Hellerstein et al., 1989). Finalement, nous pouvons penser que les AGPI n-3, en diminuant I'inflammation systé- mique, pourraient limiter l'altération du métabolisme énergétique mitochondrial. Mais cela reste à être démontré.

\section{Conclusion}

L'inflammation et notamment les cytokines joue un rôle très important dans la dénutrition cancéreuse. Les cytokines pro-inflammatoires agissent sur l'ensemble des leviers qui conduisent à la dénutrition. Parmi les facteurs à I'origine de la dénutrition cancéreuse, I'hypothèse $d$ 'une altération de l'efficacité de la synthèse d'ATP mitochondriale et également sa relation avec I'inflammation méritent d'être explorée attentivement. L'utilisation nutritionnelle des AGPI n-3 pour lutter contre la dénutrition cancéreuse, en partie en raison de leurs effets anti-inflammatoires, semble une piste prometteuse.

\section{RÉFÉRENCES}

Argiles JM, Anker SD, Evans WJ, et al. Consensus on cachexia definitions. I Am Med Dir Assoc 2010 ; 11 : 229-30.

Baracos VE. Regulation of skeletal-muscleprotein turnover in cancer-associated cachexia. Nutrition $2000 ; 16$ : 1015-8.

Bougnoux P, Hajjaji N, Ferrasson MN, Giraudeau B, Couet C, Le Floch O. Improving outcome of chemotherapy of metastatic breast cancer by docosahexaenoic acid: a phase II trial. Br J Cancer 2009 ; 101 : 1978-85.

Busquets S, Aranda X, Ribas-Carbo M, AzconBieto J, Lopez-Soriano FJ, Argiles JM. Tumour necrosis factor-alpha uncouples respiration in isolated rat mitochondria. Cytokine 2003 ; $22: 1-4$.

Calder PC. Polyunsaturated fatty acids and inflammatory processes: New twists in an old tale. Biochimie 2009 ; 91 : 791-5.

Colomer R, Moreno-Nogueira JM, GarciaLuna PP, et al. N-3 fatty acids, cancer and cachexia: a systematic review of the literature. Br J Nutr 2007 ; 97 : 823-31.

Costelli P, Bossola M, Muscaritoli M, et al. Anticytokine treatment prevents the increase in the activity of ATP-ubiquitin- and $\mathrm{Ca}(2$ + -dependent proteolytic systems in the muscle of tumour-bearing rats. Cytokine $2002 ; 19: 1-5$.

Costelli P, Muscaritoli M, Bonetto A, et al. Muscle myostatin signalling is enhanced in experimental cancer cachexia. Eur / Clin Invest $2008 ; 38: 531-8$.

Dewey A, Baughan C, Dean T, Higgins B, Johnson I. Eicosapentaenoic acid (EPA, an omega-3 fatty acid from fish oils) for the treatment of cancer cachexia. Cochrane Database Syst Rev 2007 : CD004597.

Dewys WD, Begg C, Lavin PT, et al. Prognostic effect of weight loss prior to chemotherapy in cancer patients. Eastern Cooperative Oncology Group. Am J Med $1980 ; 69$ : 491-7.

Dumas JF, Goupille C, Julienne CM, et al. Efficiency of oxidative phosphorylation in liver mitochondria is decreased in a rat model of peritoneal carcinosis. J Hepatol 2011 ; 54 : 320-7.

Dumas JF, Goupille C, Pinault M, et al. n-3 PUFA-enriched diet delays the occurrence of cancer cachexia in rat with peritoneal carcinosis. Nutr Cancer 2010 ; 62 : 343-50.

Hellerstein MK, Meydani SN, Meydani M, Wu K, Dinarello CA. Interleukin-1-induced anorexia in the rat. Influence of prostaglandins. J Clin Invest 1989 ; 84 : 228-35.

Jho DH, Babcock TA, Tevar R, Helton WS, Espat NJ. Eicosapentaenoic acid supplementation reduces tumor volume and attenuates cachexia in a rat model of progressive nonmetastasizing malignancy. JPEN / Parenter Enteral Nutr $2002 ; 26: 291-7$.

Lai V, George J, Richey L, et al. Results of a pilot study of the effects of celecoxib on cancer cachexia in patients with cancer of the head, neck, and gastrointestinal tract. Head Neck $2008 ; 30: 67-74$.

Langstein HN, Doherty GM, Fraker DL, Buresh CM, Norton JA. The roles of gamma-interferon and tumor necrosis factor alpha in an experimental rat model of cancer cachexia. Cancer Res 1991 ; 51 : 2302-6.

Llovera M, Carbo N, Lopez-Soriano J, et al. Different cytokines modulate ubiquitin gene expression in rat skeletal muscle. Cancer Lett $1998 ; 133: 83-7$.

Lopez-Soriano J, Llovera M, Carbo N, GarciaMartinez C, Lopez-Soriano FJ, Argiles JM. Lipid metabolism in tumour-bearing mice: studies with knockout mice for tumour necrosis factor receptor 1 protein. Mol Cell Endocrinol 1997 ; 132 : 93-9.

Lundholm K, Gelin J, Hyltander A, et al. Antiinflammatory treatment may prolong survival in undernourished patients with metastatic solid tumors. Cancer Res 1994 ; 54 : 5602-6.

Lundholm K, Daneryd P, Korner U, Hyltander A, Bosaeus I. Evidence that long-term COXtreatment improves energy homeostasis and body composition in cancer patients with progressive cachexia. Int / Oncol 2004 ; 24 : 505-12.

Mantovani G, Maccio A, Madeddu C, et al. Phase II nonrandomized study of the efficacy and safety of COX-2 inhibitor celecoxib on patients with cancer cachexia. / Mol Med $2010 ; 88: 85-92$. 
Morley JE, Thomas DR, Wilson MM. Cachexia: pathophysiology and clinical relevance. Am J Clin Nutr 2006 ; 83 : 735-43.

Muscaritoli M, Anker SD, Argiles J, et al. Consensus definition of sarcopenia, cachexia and pre-cachexia: joint document elaborated by Special Interest Groups (SIG) "cachexiaanorexia in chronic wasting diseases" and "nutrition in geriatrics". Clin Nutr $2010 ; 29$ : 154-9.

$\mathrm{Ng}$ EH, Lowry SF. Nutritional support and cancer cachexia. Evolving concepts of mechanisms and adjunctive therapies. Hematol Oncol Clin North Am 1991 ; 5 : 161-84.

Perumal SS, Shanthi P, Sachdanandam P. Energy-modulating vitamins - a new combinatorial therapy prevents cancer cachexia in rat mammary carcinoma. Br J Nutr 2005 ; 93 : 901-9.

Pfitzenmaier J, Vessella R, Higano CS, Noteboom JL, Wallace DJr, Corey E. Elevation of cytokine levels in cachectic patients with prostate carcinoma. Cancer 2003 ; 97 : 1211-6.

Ramos EJ, Middleton FA, Laviano A, et al. Effects of omega-3 fatty acid supplementation on tumor-bearing rats. J Am Coll Surg $2004 ; 199$ : 716-23.

Rolfe DF, Brown GC. Cellular energy utilization and molecular origin of standard meta- bolic rate in mammals. Physiol Rev $1997 ; 77$ : 731-58.

Samavati L, Lee I, Mathes I, Lottspeich F, Huttemann M. Tumor necrosis factor alpha inhibits oxidative phosphorylation through tyrosine phosphorylation at subunit I of cytochrome c oxidase. J Biol Chem 2008 ; $283: 21134-4$.

Scott HR, McMillan DC, Forrest LM, Brown DJ, McArdle CS, Milroy R. The systemic inflammatory response, weight loss, performance status and survival in patients with inoperable non-small cell lung cancer. $\mathrm{Br}$ J Cancer $2002 ; 87$ : 264-7.

Soda K, Kawakami M, Kashii A, Miyata M. Characterization of mice bearing subclones of colon 26 adenocarcinoma disqualifies interleukin- 6 as the sole inducer of cachexia. Jpn / Cancer Res 1994 ; 85 : 1124-30.

Strassmann G, Fong M, Kenney JS, Jacob CO. Evidence for the involvement of interleukin 6 in experimental cancer cachexia. J Clin Invest $1992 ; 89$ : 1681-4.

Tatsumi T, Matoba S, Kawahara A, et al. Cytokine-induced nitric oxide production inhibits mitochondrial energy production and impairs contractile function in rat cardiac myocytes. J Am Coll Cardiol 2000 ; 35 : 1338 46.

Tisdale MJ. Mechanisms of cancer cachexia. Physiol Rev 2009 ; 89 : 381-410.
Togni V, Ota CC, Folador A, et al. Cancer cachexia and tumor growth reduction in Walker 256 tumor-bearing rats supplemented with $\mathrm{N}-3$ polyunsaturated fatty acids for one generation. Nutr Cancer $2003 ; 46$ : 52-8.

Whitehouse AS, Smith HJ, Drake JL, Tisdale MJ. Mechanism of attenuation of skeletal muscle protein catabolism in cancer cachexia by eicosapentaenoic acid. Cancer Res 2001 ; 61 : 3604-9.

Wigmore SJ, Ross JA, Falconer JS, et al. The effect of polyunsaturated fatty acids on the progress of cachexia in patients with pancreatic cancer. Nutrition 1996 ; 12 : S27-30.

Wigmore SJ, Fearon KC, Maingay JP, Ross JA. Down-regulation of the acute-phase response in patients with pancreatic cancer cachexia receiving oral eicosapentaenoic acid is mediated via suppression of interleukin-6. Clin Sci (Lond) 1997 ; 92 : 215-21.

Zhang $\mathrm{HH}$, Halbleib M, Ahmad F, Manganiello VC, Greenberg AS. Tumor necrosis factor-alpha stimulates lipolysis in differentiated human adipocytes through activation of extracellular signal-related kinase and elevation of intracellular cAMP. Diabetes $2002 ; 51: 2929-35$.

Zhou X, Wang JL, Lu J, et al. Reversal of cancer cachexia and muscle wasting by ActRIIB antagonism leads to prolonged survival. Cell 2010 ; $142:$ 531-43. 